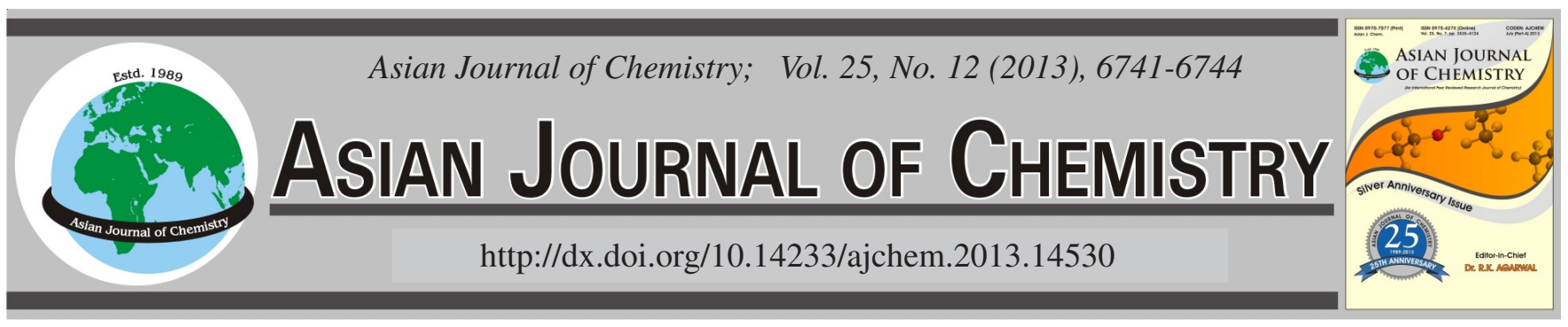

\title{
Some Element Levels in Moss Samples Collected from the Igdir-Nahhicevan International Highway, Turkey
}

\author{
Turan Özdemir ${ }^{1}$, Nevzat Batan ${ }^{2, *}$, Durali Mendil ${ }^{3}$, GöKhan Apaydin ${ }^{4}$ and Erhan Cengiz ${ }^{4}$
}

\begin{abstract}
${ }^{1}$ Department of Biology, Faculty of Science, Karadeniz Technical University, 61080 Trabzon, Turkey
${ }^{2}$ Macka Vocational School, Karadeniz Technical University, 61750 Trabzon, Turkey

${ }^{3}$ Department of Chemistry, Faculty of Science and Art, Gaziosmanpasa University, 60250 Tokat, Turkey

${ }^{4}$ Department of Physics, Faculty of Science, Karadeniz Technical University, 61080 Trabzon, Turkey
\end{abstract}

*Corresponding author: Fax: +90 46 23253195; Tel: +90 46 25123536; GSM: +90 5058061127; E-mail: nevzatbatan@ gmail.com

\begin{abstract}
Twenty-six moss and seven soil samples were collected from near the Igdir- Nahhichevan international highway in Turkey. Seventeen elements ( $\mathrm{Na}, \mathrm{Mg}, \mathrm{Al}, \mathrm{Si}, \mathrm{P}, \mathrm{S}, \mathrm{K}, \mathrm{Ca}, \mathrm{Ti}, \mathrm{Fe}, \mathrm{Cu}, \mathrm{Zn}, \mathrm{Sr}, \mathrm{Cr}, \mathrm{Mn}, \mathrm{Rb}$ and $\mathrm{Pb}$ ) were analyzed using X-ray fluorescence spectrometry. The element concentrations were found to be 1000-1862 $\mu \mathrm{g} / \mathrm{g}(\mathrm{Na}), 2174-6578 \mu \mathrm{g} / \mathrm{g}(\mathrm{Mg}), 1308-30669 \mu \mathrm{g} / \mathrm{g}(\mathrm{Al}), 11708-73710 \mu \mathrm{g} / \mathrm{g}(\mathrm{Si})$, 91-659 $\mu \mathrm{g} / \mathrm{g}(\mathrm{P}), 45-2680 \mu \mathrm{g} / \mathrm{g}(\mathrm{S}), 1123-2667 \mu \mathrm{g} / \mathrm{g}(\mathrm{K}), 5969-13997 \mu \mathrm{g} / \mathrm{g}(\mathrm{Ca}), 641-1387 \mu \mathrm{g} / \mathrm{g}(\mathrm{Ti}), 5855-17133 \mu \mathrm{g} / \mathrm{g}(\mathrm{Fe}), 23-36 \mu \mathrm{g} / \mathrm{g}$ (Cu), 10-93 $\mu \mathrm{g} / \mathrm{g}(\mathrm{Zn}), 29-114 \mu \mathrm{g} / \mathrm{g}(\mathrm{Sr}), 4-85 \mu \mathrm{g} / \mathrm{g}(\mathrm{Cr}), 17-326 \mu \mathrm{g} / \mathrm{g}(\mathrm{Mn}), 6-19 \mu \mathrm{g} / \mathrm{g}(\mathrm{Rb}), 1-14 \mu \mathrm{g} / \mathrm{g}(\mathrm{Pb})$ for mosses and 324254$402976 \mu \mathrm{g} / \mathrm{g}(\mathrm{Si}), 35347-92506 \mu \mathrm{g} / \mathrm{g}(\mathrm{Na}), 28261-76471 \mu \mathrm{g} / \mathrm{g}(\mathrm{Ca}), 3822-72123 \mu \mathrm{g} / \mathrm{g}(\mathrm{Mg}), 52731-66335 \mu \mathrm{g} / \mathrm{g}(\mathrm{Fe}), 28115-39747 \mu \mathrm{g} / \mathrm{g}$

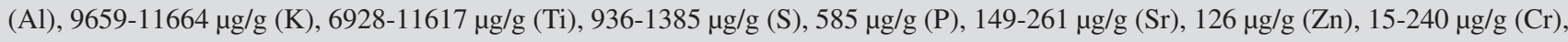
$42-180 \mu \mathrm{g} / \mathrm{g}(\mathrm{Cu}), 89 \mu \mathrm{g} / \mathrm{g}(\mathrm{Mn}), 27-46 \mu \mathrm{g} / \mathrm{g}(\mathrm{Rb})$ and $37 \mu \mathrm{g} / \mathrm{g}(\mathrm{Pb})$ for soil, respectively.
\end{abstract}

Key Words: Atmospheric deposition, Trace elements, Mosses, EDXRF, Igdir, Turkey.

\section{INTRODUCTION}

Most of the elements are essential for biological systems, whereas some metals like lead and cadmium are non-essential metals as they do not have any biological function are toxic even in traces. Iron is part of the structure of the oxygencarrying protein, hemoglobin, in the red blood cells; calcium, phosphorus and other elements constitute a significant part of the mass of teeth and bones and sodium, potassium, phosphate, sulfate, chloride and many other elements are important constituents of the fluids, both inside and outside all the body cells. Calcium in minute concentrations is necessary for normal blood clotting. Magnesium stimulates the activity of many enzymes and number of trace elements controls the contraction of muscle and the transmission of impulses by nerve cells ${ }^{1}$.

Mosses possess many properties that make them suitable bio-monitors for air pollutants ${ }^{2-4}$. They do not have real roots. So, they cannot take their nutrient from soil. Nutrient uptake from the atmosphere is promoted by their weakly developed cuticle. Their large surface-to-weight ratio improves adsorption. Slow growth rate lets them accumulate pollutants over a larger time period. Undeveloped vascular bundles allow better adsorption than vascular plants ${ }^{4}$. The attachment of the particle is affected by the size of the particle and the surface structure of the mosses ${ }^{4-6}$.

The moss monitoring technique, first introduced in Scandinavia, has shown to be very suitable for studying atmospheric deposition of heavy metals and other elements as well ${ }^{7}$. The usefulness of mosses in determining trace- and heavy-metal concentrations in different geographical areas has been discussed in many studies ${ }^{8-20}$.

Several moss species are commonly grown through IgdirNahhichevan international highway vicinity. The traffic on this highway is very high and stills the levels of metals in mosses and soils. Thus in present studies, the levels of metals in some moss and soil samples were determined by EDXRF spectrometry.

\section{EXPERIMENTAL}

For this study we have used the nine different sampling sites (Table-1). The sampling areas are shown in Fig. 1. This time ten moss species (Grimmia orbicularis, Grimmia laevigata, Syntrichia montana, Syntrichia virescens, Grimmia longirostris, Syntrichia ruralis, Homalothecium sericeum, Hypnum cupressiforme, Abietinella abietina, Grimmia ovalis) and six soil samples were collected from vicinity of roadside 


\begin{tabular}{|c|c|c|c|c|c|c|}
\hline \multicolumn{7}{|c|}{$\begin{array}{c}\text { TABLE-1 } \\
\text { DESCRIPTIONS OF THE NINE STATIONS IN IGDIR PROVINCE }\end{array}$} \\
\hline $\begin{array}{l}\text { Site } \\
\text { No }\end{array}$ & Localities & Moss samples & Nature & Latitude-longitude & $\begin{array}{l}\text { Altitude } \\
(\mathrm{m})\end{array}$ & $\begin{array}{l}\text { Collected } \\
\text { Date }\end{array}$ \\
\hline 1 & $\begin{array}{l}\text { Igdir: Karakoyunlu district, } \\
\text { Melekli town }\end{array}$ & $\begin{array}{l}\text { Grimmia orbicularis } \\
\text { Grimmia laevigata } \\
\text { Syntrichia montana } \\
\text { Soil samples }\end{array}$ & Polluted & $\begin{array}{l}39^{\circ} 57^{\prime} 30.50^{\prime \prime} \mathrm{N} \\
44^{\circ} 08^{\prime} 58.02 " \mathrm{E}\end{array}$ & 860 & 30.03 .2010 \\
\hline 2 & $\begin{array}{l}\text { Igdir: Tuzluca district } \\
\text { (between Tuzluca and } \\
\text { Kagızman district) }\end{array}$ & $\begin{array}{l}\text { Syntrichia virescens } \\
\text { Grimmia longirostris } \\
\text { Syntrichia ruralis } \\
\text { Soil samples }\end{array}$ & Polluted & $\begin{array}{l}40^{\circ} 06^{\prime} 27.77 " \mathrm{~N} \\
43^{\circ} 30^{\prime} 16.05^{\prime \prime} \mathrm{E}\end{array}$ & 1055 & 31.03 .2010 \\
\hline 3 & $\begin{array}{l}\text { Igdir: Tuzluca district, } \\
\text { Yukarıcivanlı Village }\end{array}$ & Grimmia longirostris & Unpolluted & $\begin{array}{l}40^{\circ} 00^{\prime} 31.57 " \mathrm{~N} \\
43^{\circ} 32^{\prime} 526.37 " \mathrm{E}\end{array}$ & 1882 & 16.06.2010 \\
\hline 4 & Igdir: Aralık district & $\begin{array}{l}\text { Grimmia laevigata } \\
\text { Soil samples }\end{array}$ & Polluted & $\begin{array}{l}39^{\circ} 58^{\prime} 51.89^{\prime \prime} \mathrm{N} \\
44^{\circ} 18^{\prime} 05.06^{\prime \prime} \mathrm{E}\end{array}$ & 831 & 30.03 .2010 \\
\hline 5 & Igdir: Halfeli & $\begin{array}{c}\text { Grimmia longirostris } \\
\text { Grimmia laevigata } \\
\text { Homalothecium sericeum } \\
\text { Soil saples }\end{array}$ & Unpolluted & $\begin{array}{l}39^{\circ} 51 ' 54.84 " \mathrm{~N} \\
43^{\circ} 56^{\prime} 53.53 " \mathrm{E}\end{array}$ & 1180 & 29.03.2010 \\
\hline 6 & $\begin{array}{l}\text { Igdir: Center of Tuzluca } \\
\text { district }\end{array}$ & $\begin{array}{c}\text { Syntrichia ruralis } \\
\text { Homalothecium sericeum } \\
\text { Hypnum cupressiforme } \\
\text { Soil samples }\end{array}$ & Polluted & $\begin{array}{l}40^{\circ} 03^{\prime} 09.53^{\prime \prime} \mathrm{N} \\
43^{\circ} 39^{\prime} 16.15^{\prime \prime} \mathrm{E}\end{array}$ & 1074 & 31.03 .2010 \\
\hline 7 & Igdir: Alibeyköy village & $\begin{array}{c}\text { Abietinella abietina } \\
\text { Grimmia ovalis } \\
\text { Syntrichia montana } \\
\text { Hypnum cupressiforme } \\
\text { Homalothecium sericeum } \\
\text { Soil samples }\end{array}$ & Unpolluted & $\begin{array}{l}39^{\circ} 48^{\prime} 50.92 " \mathrm{~N} \\
43^{\circ} 55^{\prime} 57.88^{\prime \prime} \mathrm{E}\end{array}$ & 1850 & 29.03 .2010 \\
\hline 8 & $\begin{array}{l}\text { Igdir: Tuzluca district, } \\
\text { Taşköprü Village }\end{array}$ & $\begin{array}{c}\text { Syntrichia virescens } \\
\text { Homalothecium sericeum } \\
\text { Hypnum cupressiforme }\end{array}$ & Polluted & $\begin{array}{l}39^{\circ} 52^{\prime} 07.40^{\prime \prime} \mathrm{N} \\
43^{\circ} 28^{\prime} 57.94^{\prime \prime} \mathrm{E}\end{array}$ & 2134 & 14.06 .2010 \\
\hline 9 & $\begin{array}{l}\text { Igdir: Korhan High Plateau, } \\
\text { Agrı Mountain }\end{array}$ & $\begin{array}{c}\text { Grimmia ovalis } \\
\text { Hypnum cupressiforme } \\
\text { Homalothecium sericeum } \\
\text { Syntrichia ruralis } \\
\end{array}$ & Unpolluted & $\begin{array}{l}39^{\circ} 47^{\prime} 11.09^{\prime \prime} \mathrm{N} \\
44^{\circ} 16^{\prime} 06.73^{\prime \prime} \mathrm{E}\end{array}$ & 1904 & 17.06.2010 \\
\hline
\end{tabular}

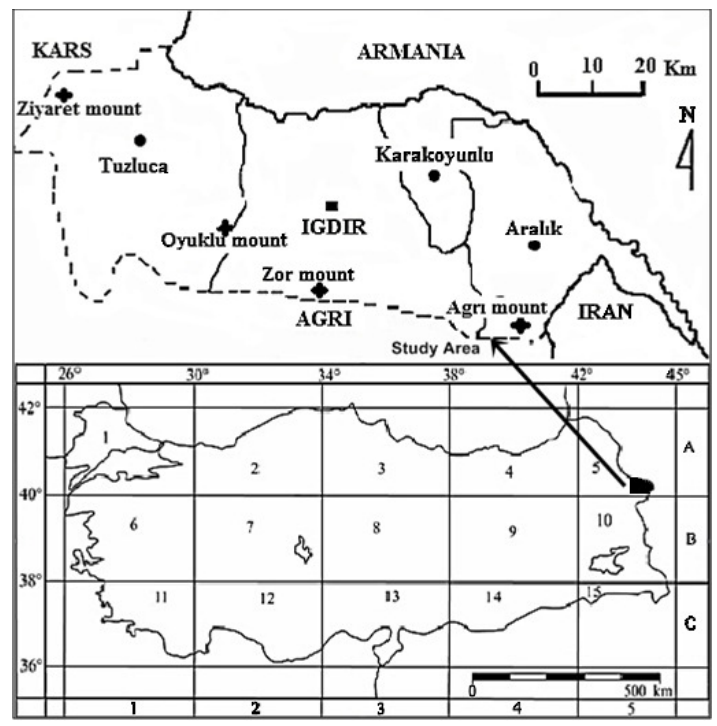

Fig. 1. Geographical location of the research area

from Igdir-Nahhichevan (5-100 meters away) during 2010. The samples were dried at $105{ }^{\circ} \mathrm{C}$ for $24 \mathrm{~h}$. Dried samples were grinded in a spex mill then the powder obtained was sieved using a 400 mesh sieve and then stirred for $20 \mathrm{~min}$ to obtain a well-mixed sample and stored in pre-cleaned polyethylene bottles until analysis. A five tone hydraulic press was used to compress the sample powder into a solid thick pellet of $40 \mathrm{~mm}$ diameter using a boric acide $\left(\mathrm{H}_{3} \mathrm{BO}_{3}\right.$-powder $)$ as a protective cover.

The elements concentration of the samples were determined using a Skyray EDX 3600B spectrometer equipped with an Oxford $\mathrm{Rh}$ anode X-ray tube (the spectrometer has a SSD high resolution detector having $145 \pm 5 \mathrm{eV}$ energy resolutions and this spectrometer is capable of $0.05 \%$ measurement precision. Besides, 24 elements can be analyzed simultaneously using it).

\section{RESULTS AND DISCUSSION}

All element concentrations were determined on a dry weight as $\mu \mathrm{g} / \mathrm{g}$. The relative standard deviations were less than $10 \%$ for all elements. t-test was used in this study $(p<0.05)$. The mean concentrations of $\mathrm{Na}, \mathrm{Mg}, \mathrm{Al}, \mathrm{Si}, \mathrm{P}, \mathrm{S}, \mathrm{K}, \mathrm{Ca}, \mathrm{Ti}, \mathrm{Fe}$, $\mathrm{Cu}, \mathrm{Zn}, \mathrm{Sr}, \mathrm{Cr}, \mathrm{Mn}, \mathrm{Rb}$ and $\mathrm{Pb}$ in moss and soil samples are given in Tables 2 and 3. The order of levels of elements in moss samples were determined as $\mathrm{Si}>\mathrm{Al}>\mathrm{Fe}>\mathrm{Ca}>\mathrm{Mg}>$ $\mathrm{Na}>\mathrm{S}>\mathrm{K}>\mathrm{Ti}>\mathrm{P}>\mathrm{Mn}>\mathrm{Sr}>\mathrm{Zn}>\mathrm{Cr}>\mathrm{Cu}>\mathrm{Rb}>\mathrm{Pb}$.

Among the elements Fe $(1713 \mu \mathrm{g} / \mathrm{g}), \mathrm{Cu}(36 \mu \mathrm{g} / \mathrm{g}), \mathrm{Mn}$ $(326 \mu \mathrm{g} / \mathrm{g}), \mathrm{Zn}(93 \mu \mathrm{g} / \mathrm{g})$ and $\mathrm{Cr}(85 \mu \mathrm{g} / \mathrm{g})$ higher concentrations on the traffic roadside were than control areas. Levels of, iron, copper, manganese, zinc and chromium showed highest concentrations on the traffic roadside. Copper, zinc and chromium had approximate similar concentrations on the traffic roadside and control areas. The concentration of trace 


\section{TABLE-2}

CONCENTRATIONS OF ELEMENTS IN MOSS SPECIES $(\mu \mathrm{g} / \mathrm{g})$

\begin{tabular}{|c|c|c|c|c|c|c|c|c|c|c|c|c|c|c|c|c|c|c|c|}
\hline No & Moss species & Site & $\mathrm{Na}$ & $\mathrm{Mg}$ & $\mathrm{Al}$ & $\mathrm{Si}$ & $\mathrm{P}$ & $\mathrm{S}$ & $\mathrm{K}$ & $\mathrm{Ca}$ & $\mathrm{Ti}$ & $\mathrm{Fe}$ & $\mathrm{Cu}$ & $\mathrm{Zn}$ & $\mathrm{Sr}$ & $\mathrm{Cr}$ & $\mathrm{Mn}$ & $\mathrm{Rb}$ & $\mathrm{Pb}$ \\
\hline 1 & Grimmia orbicularis & 1 & 1152 & 5001 & 10482 & 53990 & 249 & 1341 & 1866 & 9201 & 1235 & 15301 & 25 & 42 & 65 & 38 & 17 & 12 & 3 \\
\hline 2 & Grimmia laevigata & 1 & 1237 & 4748 & 30669 & 73710 & 507 & 854 & 2667 & 9637 & 1330 & 17133 & 27 & 23 & 50 & 49 & 80 & 13 & 3 \\
\hline 3 & Syntrichia montana & 1 & 1316 & 3449 & 17762 & 42002 & 600 & 1280 & 2406 & 9978 & 1032 & 13604 & 27 & 33 & 52 & 37 & 123 & 10 & 3 \\
\hline 4 & Syntrichia virescens & 2 & 1158 & 4887 & 15069 & 69671 & 214 & 1300 & 2334 & 10804 & 1387 & 16306 & 24 & 21 & 81 & 24 & 29 & 11 & 1 \\
\hline 5 & Grimmia longirostris & 3 & 1540 & 5405 & 6006 & 74644 & 225 & 1730 & 2559 & 6106 & 1154 & 12752 & 26 & 29 & 57 & 7 & 99 & 16 & 9 \\
\hline 6 & Grimmia laevigata & 4 & 1684 & 6578 & 1308 & 40405 & 432 & 2000 & 1708 & 7574 & 1150 & 13298 & 25 & 71 & 57 & 26 & 41 & 7 & 8 \\
\hline 7 & Grimmia longirostris & 5 & 1348 & 4610 & 1308 & 42911 & 196 & 2103 & 2182 & 7628 & 1318 & 15305 & 28 & 79 & 67 & 30 & 103 & 14 & 14 \\
\hline 8 & Grimmia laevigata & 5 & 1648 & 5859 & 1308 & 47968 & 91 & 1705 & 2124 & 6952 & 1338 & 15159 & 27 & 47 & 55 & 34 & 20 & 12 & 8 \\
\hline 9 & Homalothecium sericeum & 6 & 1593 & 5051 & 1308 & 32803 & 312 & 1783 & 2004 & 13928 & 1007 & 11738 & 25 & 60 & 90 & 36 & 131 & 12 & 8 \\
\hline 10 & Syntrichia ruralis & 6 & 1459 & 5695 & 1308 & 29607 & 290 & 1573 & 2091 & 9731 & 1088 & 12585 & 28 & 72 & 65 & 16 & 242 & 19 & 11 \\
\hline 11 & Hypnum cupressiforme & 6 & 1745 & 5329 & 1308 & 28517 & 390 & 1384 & 2093 & 10577 & 974 & 11177 & 29 & 72 & 55 & 45 & 167 & 10 & 9 \\
\hline 12 & Grimmia longirostris & 2 & 1356 & 5190 & 9813 & 38955 & 202 & 1306 & 1262 & 12119 & 1083 & 15200 & 25 & 18 & 86 & 39 & 17 & 6 & 3 \\
\hline 13 & Syntrichia ruralis & 2 & 1371 & 5859 & 1308 & 34470 & 343 & 1522 & 2032 & 13997 & 1146 & 16469 & 27 & 18 & 114 & 33 & 145 & 12 & 1 \\
\hline 14 & Abietinella abietina & 7 & 1418 & 2402 & 11113 & 27899 & 659 & 926 & 2523 & 9569 & 864 & 8051 & 32 & 44 & 36 & 59 & 253 & 9 & 1 \\
\hline 15 & Grimmia ovalis & 7 & 1377 & 4610 & 18208 & 58815 & 364 & 1408 & 2083 & 7418 & 1141 & 10916 & 29 & 55 & 45 & 4 & 96 & 11 & 6 \\
\hline 16 & Syntrichia montana & 7 & 1535 & 3676 & 14308 & 36768 & 611 & 1002 & 2275 & 7703 & 930 & 10124 & 29 & 77 & 60 & 6 & 168 & 12 & 6 \\
\hline 17 & Hypnum cupressiforme & 7 & 1000 & 2427 & 9832 & 39617 & 185 & 1640 & 1825 & 5969 & 804 & 8300 & 26 & 10 & $2 \varepsilon$ & 4 & 61 & 9 & 2 \\
\hline 18 & Homalotheciu & 7 & 116 & 2174 & 3611 & 24990 & 313 & 45 & 2065 & 7510 & 740 & 7124 & 29 & 29 & & 4 & 115 & 9 & 1 \\
\hline 19 & Syntrichia virescens & 8 & 170 & 3600 & 6508 & 16937 & 354 & 2680 & 1123 & 10307 & 728 & 5855 & 36 & 32 & 33 & 85 & 326 & 9 & 3 \\
\hline 20 & Homalothecium sericeum & 8 & 1862 & 4433 & 2886 & 11708 & 284 & 45 & 2091 & 7904 & 641 & 6819 & 33 & 93 & 29 & 46 & 251 & 9 & 3 \\
\hline 21 & Grimmia ovalis & 9 & 1739 & 3916 & 24150 & 50943 & 488 & 570 & 2254 & 7326 & 1068 & 11863 & 29 & 69 & 63 & 4 & 237 & 13 & 3 \\
\hline 22 & Hypnuт cupressiforme & 8 & 1432 & 2212 & 5226 & 15908 & 165 & 45 & 1912 & 9314 & 779 & 7706 & 30 & 76 & 37 & 52 & 278 & 9 & 9 \\
\hline 23 & Hypnum cupressiforme & 9 & 1371 & 4988 & 6062 & 42421 & 379 & 1254 & 2176 & 7896 & 991 & 10012 & 28 & 55 & 36 & 22 & 106 & 11 & 5 \\
\hline 24 & Homalothecium sericeum & 9 & 1511 & 3121 & 9423 & 26986 & 362 & 102 & 2066 & 7690 & 673 & 6208 & 32 & 64 & 29 & 27 & 202 & 6 & 6 \\
\hline 25 & Syntrichia ruralis & 9 & 1193 & 3954 & 13008 & 43160 & 252 & 45 & 1932 & 6519 & 1096 & 12170 & 28 & 63 & 59 & 4 & 134 & 13 & 8 \\
\hline 26 & Syntrichia princeps & 9 & 1102 & 2528 & 4409 & 32376 & 201 & 1027 & 1827 & 6439 & 1043 & 10406 & 23 & 17 & 76 & 4 & 17 & 13 & 1 \\
\hline
\end{tabular}

TABLE-3

CONCENTRATIONS OF ELEMENTS IN SOIL SAMPLES $(\mu \mathrm{g} / \mathrm{g})$

\begin{tabular}{cccccccccccccccccccc}
\hline Samples & Sites & $\mathrm{Na}$ & $\mathrm{Mg}$ & $\mathrm{Al}$ & $\mathrm{Si}$ & $\mathrm{P}$ & $\mathrm{S}$ & $\mathrm{K}$ & $\mathrm{Ca}$ & $\mathrm{Ti}$ & $\mathrm{Fe}$ & $\mathrm{Cu}$ & $\mathrm{Zn}$ & $\mathrm{Sr}$ & $\mathrm{Cr}$ & $\mathrm{Mn}$ & $\mathrm{Rb}$ & $\mathrm{Pb}$ \\
\hline Soil & 1 & 89984 & 58112 & 33421 & 397267 & 230 & 936 & 11131 & 45173 & 7184 & 62098 & 180 & 171 & 149 & 188 & 44 & 38 & 25 \\
Soil & 2 & 92506 & 72123 & 30561 & 324254 & 218 & 1385 & 10335 & 76471 & 7690 & 66335 & 144 & 145 & 253 & 240 & 89 & 28 & 8 \\
Soil & 4 & 46835 & 53961 & 28115 & 369166 & 585 & 1003 & 10372 & 38949 & 6928 & 52731 & 122 & 128 & 188 & 164 & 49 & 36 & 5 \\
Soil & 5 & 89143 & 51452 & 36112 & 402976 & 218 & 1184 & 11304 & 35099 & 7183 & 57878 & 105 & 126 & 173 & 129 & 38 & 27 & 26 \\
Soil & 6 & 60564 & 48079 & 31814 & 384513 & 218 & 1143 & 11664 & 45177 & 7066 & 55519 & 163 & 208 & 176 & 122 & 74 & 46 & 37 \\
Soil & 7 & 35347 & 38220 & 39754 & 365441 & 218 & 1328 & 9659 & 28261 & 11617 & 63536 & 42 & 154 & 261 & 15 & 38 & 36 & 5 \\
\hline
\end{tabular}

metals in the samples are depended on moss species. For example, the high element accumulation levels in the species were found in Grimmia laevigata for Si $(73710 \mu \mathrm{g} / \mathrm{g}), \mathrm{Al}$ (30669 $\mu \mathrm{g} / \mathrm{g}), \mathrm{Fe}(17133 \mu \mathrm{g} / \mathrm{g}), \mathrm{Mg}(4433 \mu \mathrm{g} / \mathrm{g}), \mathrm{K}(2667 \mu \mathrm{g} /$ g), $\mathrm{Pb}(14 \mu \mathrm{g} / \mathrm{g})$, Syntrichia ruralis for Ca $(13997 \mu \mathrm{g} / \mathrm{g}), \mathrm{Sr}$ $(114 \mu \mathrm{g} / \mathrm{g}), \mathrm{Rb}(19 \mu \mathrm{g} / \mathrm{g}), \mathrm{Sr}(114 \mu \mathrm{g} / \mathrm{g})$, Syntrichia virescens for $\mathrm{Mn}(326 \mu \mathrm{g} / \mathrm{g}), \mathrm{Cr}(85 \mu \mathrm{g} / \mathrm{g}), \mathrm{Cu}(36 \mu \mathrm{g} / \mathrm{g}), \mathrm{Ti}(1387 \mu \mathrm{g} / \mathrm{g})$, $\mathrm{S}(2680 \mu \mathrm{g} / \mathrm{g})$, Homalothecium sericeum for $\mathrm{Na}(1862 \mu \mathrm{g} / \mathrm{g})$, $\mathrm{Zn}(93 \mu \mathrm{g} / \mathrm{g})$ and Abietinella abietina for P $(659 \mu \mathrm{g} / \mathrm{g})$, respectively.

The element concentrations in soil samples were found to be 324254-402976, 35347-92506, 28261-76471, 382272123, 52731-66335, 28115-39747, 9659-11664, 692811617, 936-1385, 585, 149-261, 126, 15-240, 42-180, 89, 27-46 and $37 \mu \mathrm{g} / \mathrm{g}$ for Si, Na, Ca, Mg, Fe, Al, K, Ti, S, P, Sr, $\mathrm{Zn}, \mathrm{Cr}, \mathrm{Cu}, \mathrm{Mn}, \mathrm{Rb}$ and $\mathrm{Pb}$, respectively. The order of levels of metals in soil samples as $\mathrm{Si}>\mathrm{Na}>\mathrm{Ca}>\mathrm{Mg}>\mathrm{Fe}>\mathrm{Al}>\mathrm{K}$ $>\mathrm{Ti}>\mathrm{S}>\mathrm{P}>\mathrm{Sr}>\mathrm{Zn}>\mathrm{Cr}>\mathrm{Cu}>\mathrm{Mn}>\mathrm{Rb}>\mathrm{Pb}$.

Iron is a relatively abundant element in the universe. The main iron emission sources can be coal-burning and intensive traffic $^{21,22}$. The lowest and highest iron concentrations were found to be $5855 \mu \mathrm{g} / \mathrm{g}$ in Syntrichia virescens and $17133 \mu \mathrm{g} / \mathrm{g}$ in Grimmia laevigata. Our results were higher than those reported earlier ${ }^{13,20,23-28}$. Aluminium concentration was found $30669 \mu \mathrm{g} / \mathrm{g}$ in Grimmia laevigata. Aluminium is the second high concentration values after $\mathrm{Si}$ in moss samples. Aluminium values have been reported lower for different moss species by Giordano et al. and Barandowski et al. ${ }^{26,27}$.

Zinc is involved in the metabolism of energy, proteins, carbohydrates, lipids and nucleic acids and is an essential element for tissue accretion ${ }^{1}$. Zinc concentrations were found as $10-93 \mu \mathrm{g} / \mathrm{g}$ in moss samples. Zinc average values are in good agreement with literature values as $14-203 \mu \mathrm{g} / \mathrm{g}^{27}$.

Average chromium concentration ranged $4-85 \mu \mathrm{g} / \mathrm{g}$ in moss samples. Chromium values are higher than literature values for Serbia, Romania, Bulgaria and quite higher than Norway, Finland, North Spain, Hungary, Poland, France, Germany, Netherlands and Czech Republic ${ }^{13,20,24,26-28}$. Cr the other emission sources are intensive traffic, coal-fired power plant and coal-mining Works ${ }^{29,30}$.

Copper concentration was found $23-36 \mu \mathrm{g} / \mathrm{g}$ in the moss samples. Our copper results were higher than literature values ${ }^{20,24,31}$. Giordona et al., were reported higher values than ours $^{26}$. Copper mainly originates from the metal industry, mining, coal-fired plants, traffic, copper-containing fungicides and fertilizers used by agriculture and even from soil ${ }^{21}$. 
Copper compounds are widely used in the environment as fertilizers and nutritional supplements and, because of their microbicidal properties, as fungicides, algaecides, insecticides and wood preservatives ${ }^{1}$.

The maximum lead concentration was found as $14 \mu \mathrm{g} / \mathrm{g}$ in unpolluted area. The adding of lead to the petrol increases the concentration of lead. In literature, the high lead concentration has been reported in the samples of high-density traffic $\operatorname{areas}^{32}$. Our lead value is lower than literature values ${ }^{13,16,24,26-28}$. Combustion of leaded fuel is still a main source of lead pollution, other sources such as metal production, motor vehicles, soils, coal combustions and mining ${ }^{28}$.

Average $\mathrm{Si}, \mathrm{Ca}, \mathrm{Na}, \mathrm{S}, \mathrm{K}, \mathrm{Ti}, \mathrm{P}, \mathrm{Mn}, \mathrm{Sr}$ and $\mathrm{Rb}$ concentrations were found to be 11708-73710, 5969-13997, 1000$1862,2680,1123-2667,91-1387,14,326,114$ and 6-19 $\mu \mathrm{g} / \mathrm{g}$ in moss samples, respectively.

Average $\mathrm{Si}, \mathrm{Na}, \mathrm{Ca}, \mathrm{Mg}, \mathrm{Fe}, \mathrm{Al}, \mathrm{K}, \mathrm{Ti}, \mathrm{S}, \mathrm{P}, \mathrm{Sr}, \mathrm{Zn}, \mathrm{Cr}, \mathrm{Cu}$, $\mathrm{Mn}, \mathrm{Rb}$ and $\mathrm{Pb}$ were found to be 324254-402976, 3534792506, 28261-76471, 3822-72123, 52731-66335, 2811539747, 9659-11664, 6928-11617, 936-1385, 585, 149-261, $126,15-240,42-180,89,27-46$ and $37 \mu \mathrm{g} / \mathrm{g}$, in soil samples, respectively. In this study, the moss samples were found to be the best bioindicator for all elements.

\section{ACKNOWLEDGEMENTS}

The authors gratefully acknowledged the financial supports of the Turkish Scientific and Technical Research Council (TBAG-108T566 coded project).

\section{REFERENCES}

1. J.J. Strain and K.D. Cashman, Minerals and Trace Elements, in: Introduction to Human Nutrition, edn. 2, Ch. 9, pp. 188-237 (2009).

2. P.C. Onianwa, Environ. Monit. Assess., 71, 13 (2001).

3. H.G. Zeichmeister, K. Grodzinska and G. Szarek-Lukaszewska, In eds. B.A. Markert, A.M. Breure and H.G. Zeichmeister, Bryophytes, Elsevier, Oxford, pp. 329-375 (2003).

4. S. Chakrabortty and G.T. Paratkar, Aerosol Air Quality Res., 6, 247 (2006).

5. D.H. Brown and J.W. Bates, Bot. J. Linnean Soc., 104, 129 (1990).

6. G. Tyler, Bot. J. Linnean Soc., 104, 231 (1990).

7. A. Ruhling and G. Taylor, Environ. Pollut., 131, 417 (2004).

8. U. Herpin, B. Markert, V. Weckert, J. Berlecamp, K. Friese, U. Siewers and H. Lieth, Sci. Total Environ., 205, 1 (1997).
9. D. Weiss, W. Shotyk, J.D. Kramers and M. Gloor, Atmos. Environ., 33, 3751 (1999).

10. J.A. Fernandez and A. Carbelleria, Environ. Pollut., 114, 431 (2001).

11. A. Carballeira, J.A. Couto and J.A. Fernandez, Water Air Soil Pollut., 133, 235 (2002).

12. J. Sardans and J. Penuelas, Chemosphere, 60, 1293 (2005).

13. H. Harmens, D.A. Norris, E. Steinnes, E. Kubin, J. Piispanen, R. Alber, Y. Aleksiayenak, O. Blum, M. Coskun, M. Dam, L. De Temmerman, J.A. Fernandez, M. Frolova, M. Frontasyeva, L. Gonzales-Miqueo, K. Grodzinska, Z. Jeran, S. Korzekwa, M. Krmar, K. Kvietkus, S. Leblond, S. Liiv, S.H. Magnusson, B. Mankovska, R. Pesch, A. Rühling, J.M. Santamaria, W. Schröder, Z. Spiric, I. Suchara, L. Thöni, V. Urumov, L. Yurukova and H.G. Zechmeister, Environ. Pollut., 158, 3144 (2010).

14. T. Özdemir, G. Apaydin, D. Mendil, V.N. Bulut, E. Cengiz, A. Gündogdu and V. Aylikci, Asian J. Chem., 22, 346 (2010).

15. M. Tretiach, E. Pittao, P. Crisafulli and P. Adamo, Sci. Total Environ., 408, 6291 (2010)

16. A. Ares, J.A. Fernandez, J.R. Aboal and A. Carballeira, Ecotoxicol. Environ. Safety, 74, 533 (2011).

17. Z.M. Migaszewski, P.J. Lamothe, J.G. Crock, A. Galuszka and S. Dolegowska, Environ. Chem. Lett., 9, 323 (2011).

18. M.T. Boquete, J.A. Fernandez, J.R. Aboal and A. Carballeira, Environ. Exp. Botany, 72, 210 (2011).

19. M.T. Boquete, J.A. Fernandez, J.R. Aboal and A. Carballeira, Atmos. Environ., 45, 2704 (2011).

20. C. Mariet, A. Gaudry, S. Ayrault, M. Moskura, F. Derayer and N. Bernard, Environ. Monit. Assess, 174, 107 (2011).

21. A. Rühling and E. Steinnes, Atmospheric Heavy Metal Deposition in Europe 1995-1996, Nordic Council of Ministers, 15, pp. 1-67 (1998).

22. K. Namik, O. Aras and Y. Ataman, Trace Element Analiysis of Food and Diet, Importance of Trace Elements in Food, Published by the Royal Society of Chemistry, Cambridge, Ch. 1, p. 344 (2006).

23. R. Figueira, C. Sergio and A.J. Sousa, Environ. Pollut., 118, 153 (2002).

24. B. Vargha, E. Ötvös and Z. Tuba, Ann. Agric. Environ. Med., 9, 141 (2002).

25. P. Adamo, S. Giordano, S. Vingiani, R.C. Cobianchi and P. Violente, Environ. Pollut., 122, 91 (2003).

26. S. Giordano, P. Adamo, S. Sorbo and S. Vingiana, Environ. Pollut., 136, 431 (2005).

27. L. Barandovski, M. Cekova, M.V. Frontasyeva, S.S. Pavlov, T. Stafilov, E. Steinnes and V. Urumov, Environ. Monit. Assess, 138, 107 (2008).

28. G. Uyar, M. Ören, Y. Yildirim and S. Öncel, Environ. Forensics, 9, 350 (2008).

29. G. Uyar, M. Ören, Y. Yildirim and M. Ince, Fresenius Environ. Bull., 16, 182 (2007).

30. G. Uyar, M. Ören and M. Ince, Fresenius Environ. Bull., 16, 145 (2007).

31. G. Uyar, E. Avcil, M. Ören, F. Karaca and M.S. Oncel, Environ. Eng. Sci., 26, 183 (2009).

32. M. Tüzen, D. Mendil, H. Sari and E. Hasdemir, Fresenius Environ. Bull., 12, 1283 (2003). 